\title{
Earth-like planet detection with Extremely Large Telescopes: fundamental limitations
}

\author{
Cavarroc Céline ${ }^{1}$, Boccaletti Anthony ${ }^{1}$, Baudoz Pierre ${ }^{1}$, \\ Fusco Thierry ${ }^{2}$ and Rouan Daniel ${ }^{1}$ \\ ${ }^{1}$ Observatoire de Paris-Meudon, ${ }^{2}$ ONERA
}

\begin{abstract}
Direct detection and characterization of Earth-like planets from the ground is a very challenging issue. Among the projects, the Extremely Large Telescopes are very promising to improve the angular resolution and to increase the total number of collected photons. We studied this type of instruments in a very optimistic case to evaluate what level of aberrations limits fundamentally the detection. For that purpose, we considered a perfect coronagraph coupled with an extreme adaptive optics device. Even with a Strehl ratio of more than $96 \%$, it only provides a contrast of $10^{-6}-10^{-7}$ at $30 \lambda / D$. A calibration system downstream the coronagraph is therefore mandatory to reach the contrast of $10^{-10}$ between a terrestrial planet and its star in the near infra-red. We modelized a very general system taking into account dynamic aberrations left uncorrected by the adaptive optics system, static aberrations of the system and differential static aberrations due to the calibration channel. Numerical simulations demonstrate that the static aberrations are becoming very limitative and must not be neglected. Indeed, to achieve a contrast of $10^{-10}$, with common aberrations of $5 \mathrm{~nm}$ on a 100 meter telescope, the differential aberrations must be controlled at the level of 200 picometers. We also compare this speckle noise to the limitation due to the photon noise.
\end{abstract}

Keywords. instrumentation: high angular resolution, instrumentation: adaptive optics, planetary systems.

\section{Introduction}

After the discovery of more than 150 extra-solar planetary systems for ten years by indirect methods, direct imaging and characterization of Earth-like planets has became the next challenge. Extremely Large Telescopes (ELT) are promising tools to reach the required performance. Several concepts of ELT exist around the world with sizes from 30 to $100 \mathrm{~m}$. This new generation of telescopes will require complete studies to evaluate their performances. The present study takes place in this context.

The detection of Earth-like planet in the near infra-red imposes to reach a contrast of $10^{-10}$ at close angular distance to a star. For that purpose, we consider a coronagraph associated with a extreme adaptive optics system. With this system, even very performing, we may not expect a contrast larger than $10^{-6}-10^{-7}$ at $30 \lambda / D$. To enhance it, we consider a simultaneous calibration system with one channel which shows the planet whereas the other does not.

Section 2 presents the system and the hypotheses we made. Then, we demonstrate (section 3) the performance which can be reached from this system and what is limitative. Finally, in section 4 , we analyze the limitations due the photon noise and the performance of the wavefront sensor. 


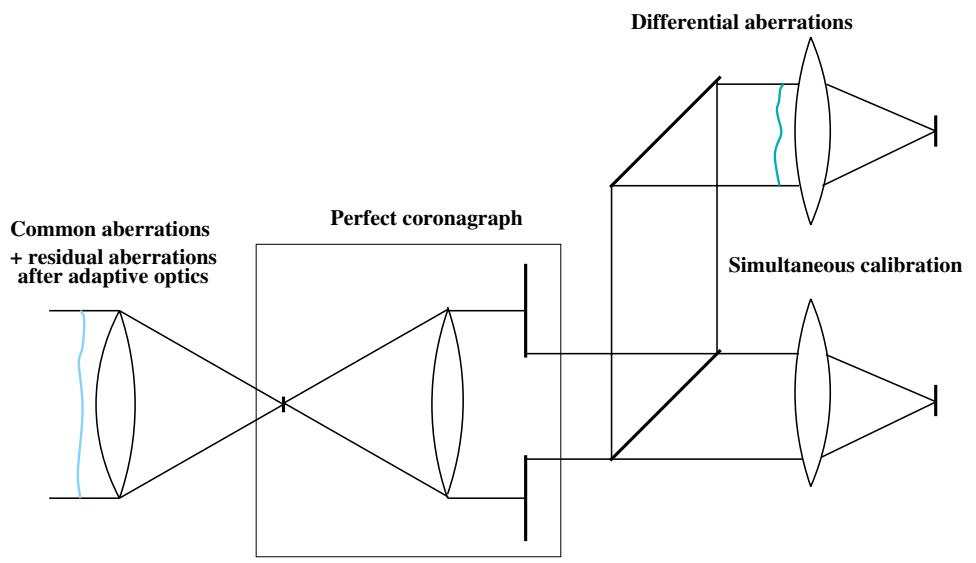

Figure 1. Generic concept of the studied system with from left to right the telescope pupil, the focal plane, the relayed optics with the Lyot stop and differential optics. The intensity distribution at a given plane is defined by the Fourier transform of the upstream plane. Phase aberrations are included in the telescope pupil and in the pupil after the beamsplitter.

\section{System}

We assume that the instrument is a one hundred meter telescope composed with a classical coronagraphic system and a calibration channel downstream the coronagraph (Fig. 1). This calibration is performed simultaneously to reduce the speckle noise. Besides we consider the perfect case in which the planet is shown in one channel and not in the other. We do not assume any particular system of calibration (several types exist using properties of the planet: its spectral properties Racine (1999), its polarization or its coherence with respect to the star).

The aim of this paper is to study only the effects of the aberrations on the performance of the system and the limitations they impose. So, we assume a monolithic entrance pupil (without segmentation) and a perfect coronagraph, i.e. a coronagraph which removes perfectly all the coherent light. It is simulated by subtracting a perfect pupil to the actuall pupil.

Meanwhile, the system is not perfect: we assume an adaptive optics (AO) system which provides a Strehl ratio of about $98 \%$. The atmospheric phase screens are generated from analytical expressions Rigaut (1998). We represent in Fig. 2 their power spectrum densities (PSD). We consider a Shack-Hartmann wavefront sensor (SHS) with 200 actuators across the pupil diameter (the choice has been made from an extrapolation from VLT$\mathrm{PF}$ ). The seeing is 0.75 arcsecond at $l a m b d a=0.5 \mu \mathrm{m}$. The imaging wavelength is 1.63 $\mu \mathrm{m}$. We assume a 5 th magnitude star and a loop frequency of $2.5 \mathrm{kHz}$. The PSD includes fitting errors, the servo-lag and the photon noise of the wavefront sensor. The aliasign is filtered in the wavefront sensor.

We also consider static aberrations of the instrument: common aberrations upstream the coronagraph and differential aberrations due to the difference of optical path between the two channels of the calibration. The classical optical aberrations are represented by a PSD with a slope in $f^{-2}$ with $f$ the spatial frequency Duparré (2002). We consider this type of PSD but with an improvement: we estimate that low frequencies can be corrected (by a better polishing or by a correction -phase diversity for instance) under a given cut-off frequency. The two PSDs are represented in Fig. 2. 


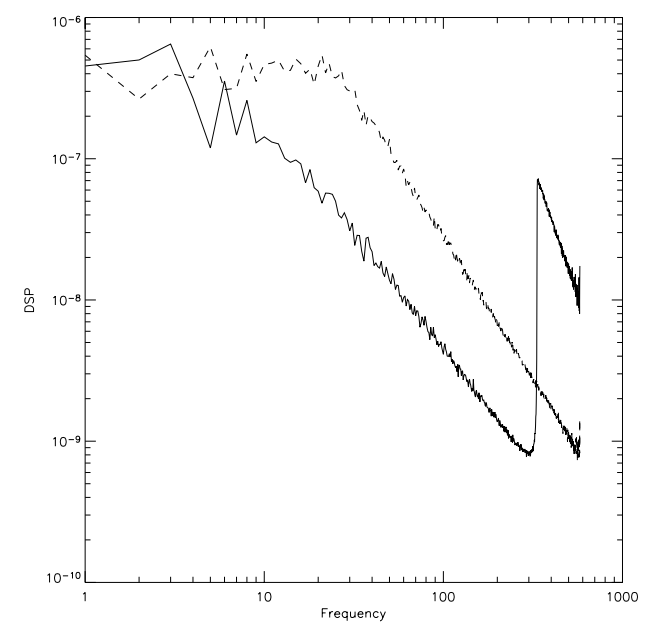

Figure 2. One run of the PSD used in our simulations: the solid line corresponds to the PSD of the residual aberrations after the AO system whereas the dashed line corresponds to the static aberrations (common and differential).

\section{Speckle noise}

First of all, we will only study the speckle noise and the limitation it imposes on the detectability level. This detectability corresponds to the angular standard deviation.

\subsection{Gain brought by the differential imaging}

We compare the initial detectability level to the one of the image after subtraction for respectively a short and a long exposures. This long exposure is made by adding a few hundreds of images (about 500) obtained from decorrelated phase screens while static aberrations remain unchanged.

Fig. 3 compares these detectability levels at $5 \sigma$ assuming a static common path aberration of $5 \mathrm{~nm}$ and a differential aberration of $0.2 \mathrm{~nm}$.

The instantaneous detectability is impressed by the AO correction: for a short exposure it limits the detectability, even after subtraction. But by subtracting, we almost gain a factor 1000 for a short exposure.

We note, in Fig. 3, that the final level of detectability for a long exposure is only set by the static aberrations (both common and differential). Indeed, the residual aberrations after AO average azimuthally and do not influence the standard deviation anymore. A more detailed study of this limitation is made in Cavarroc (2005). With common static aberrations of $5 \mathrm{~nm}$ and differential ones of $0.2 \mathrm{~nm}$ the limit of detectability is about $10^{-10}$ at 0.1 " which is enough to detect Earth-like planets but which are also very challenging values.

\subsection{Influence of static aberrations}

Because the level is set by static aberrations, the shape of the PSF is crucial: if we choose a cut-off frequency lower (or a pure slope in $f^{-2}$ ) with the same error budget, the final level will be lower at high frequencies but higher in the region of interest.

Fig. 4 shows the influence of the amplitude of the different types of static aberrations for an angular separation of $30 \lambda / D(0.1$ " for a diameter of $100 \mathrm{~m})$. The effect of the common and differential aberrations is clearly different: the detectability varies linearly with the differential aberrations and quadratically with common aberrations. 


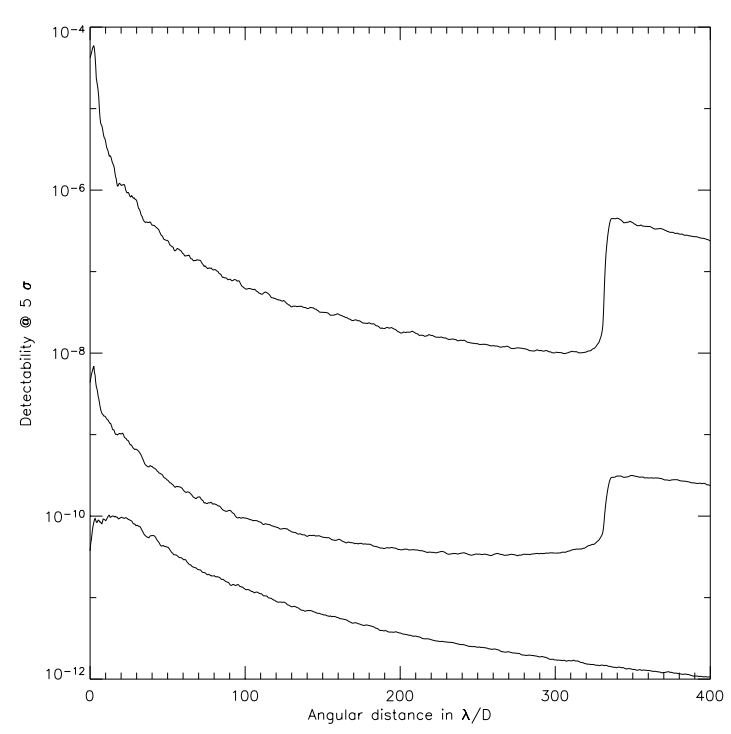

Figure 3. Detectability at $5 \sigma$. The upper curve corresponds to the detectability for a short exposure in a direct imaging mode, the middle curve for a short exposure after subtraction and the lower for a long exposure after subtraction.

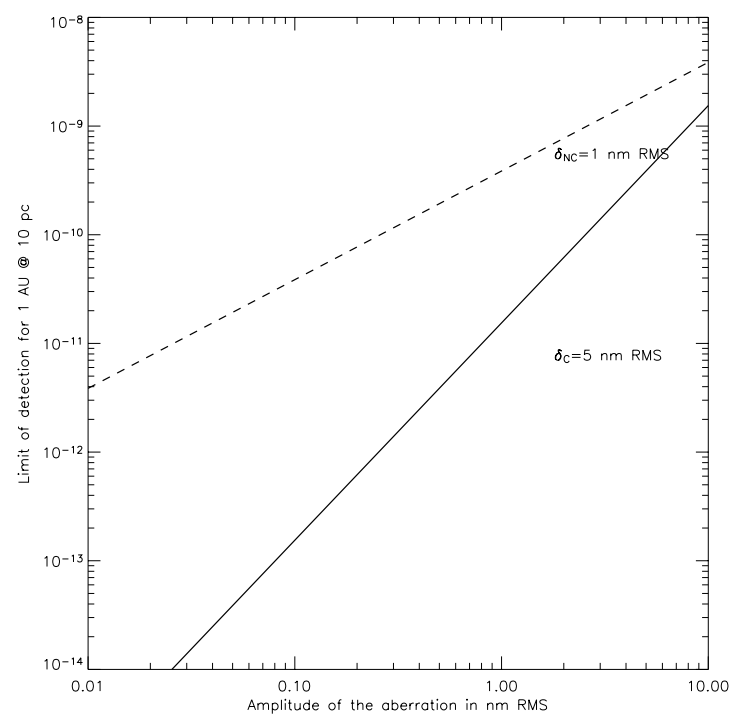

Figure 4. Detectability at $5 \sigma$ for an angular separation of $30 \lambda / D$ corresponding to $1 \mathrm{AU}$ at $10 \mathrm{pc}$ for a long exposure. The solid line stands for the impact of the common static aberration when fixing the differential aberrations and conversely for the dashed line.

Thus, to reach the desired contrast of $10^{-10}$ at 0.1 ", for a common aberration of $5 \mathrm{~nm}$, we have to control differential aberrations to $0.2 \mathrm{~nm}$, which is the assumption we used in this study, and, conversely, for differential aberrations of $1 \mathrm{~nm}$ the common aberrations must be reduced under $0.3 \mathrm{~nm}$ RMS.

Common aberrations, i.e. aberrations upstream the coronagraph, are dominant and must be reduced as far as possible. 

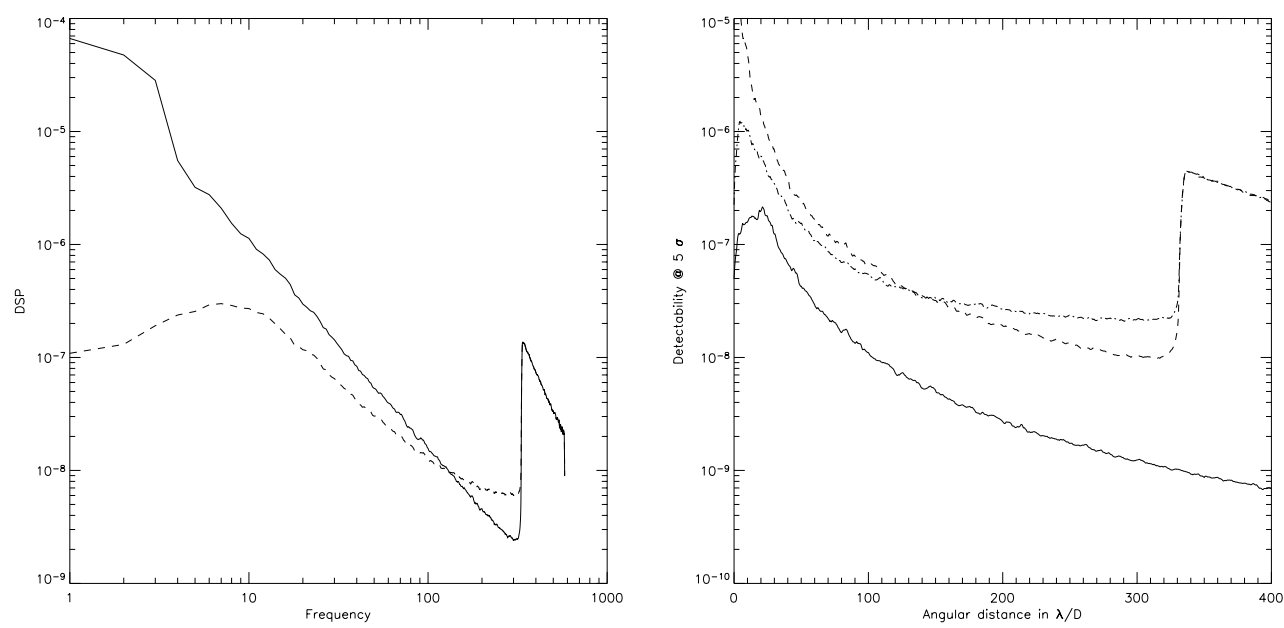

Figure 5. Left figure compares smoothed PSD of AO defects for a SHS (solid line) and a pyramidal sensor (dashed line). Right one compares the detectability level at $5 \sigma$ of the images: the solid line corresponds to the level impressed only by static aberrations, the dashed line to the level obtained with a Shack-Hartmann sensor and the dash-dotted line to the level obtained with a pyramidal sensor.

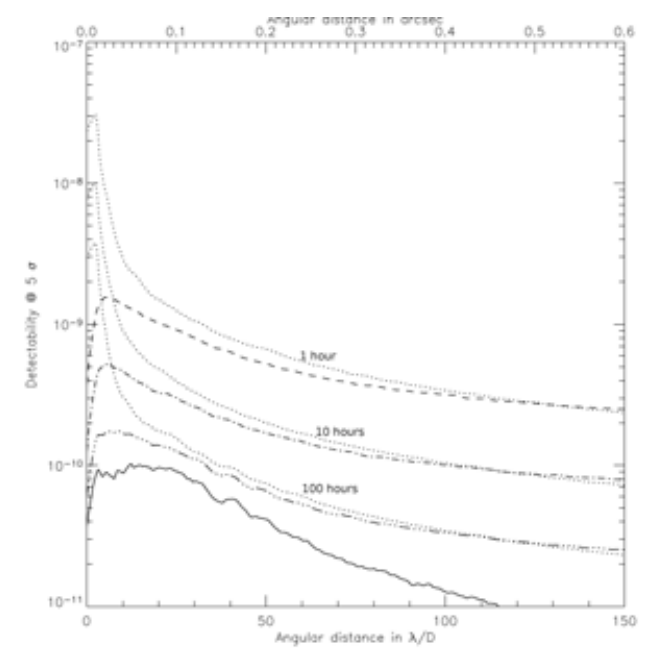

Figure 6. Radial profile of the detectability at $5 \sigma$ for different exposure times. The lower curve corresponds to the theoretical limit for an infinitely long exposure. Dotted line corresponds to the level of detectability for a Shack-Hartmann sensor whereas other lines stand for a pyramidal wavefront sensor.

\section{Photon noise}

Until now, the whole study was led assuming a Shack Hartmann sensor and considering only the speckle noise. This section is dedicated to the study of the limitation of detectability due to photon noise and the interest of using a pyramidal sensor. It is indeed important to know if the level of the contrast set by static aberrations is achievable in a reasonable amount of time. For that purpose, we assume a G2V star of magnitude 5 located at $10 \mathrm{pc}$, considering a spectral resolution of $\lambda / \Delta \lambda=5$ and a total transmission of $5 \%$. 
Figure 5 compares the Shack-Hartmann and the pyramidal sensors with the same parameters: until $150 \lambda / D$, the latter is more interesting than the former because it better corrects these low frequencies.

Figure 6 gives the limit of detectability for several exposure times from 1 to 100 hours for a system with a Shack-Hartmann sensor or with a pyramidal sensor and compares these levels to the limit set by static aberrations. By increasing the exposure time, the detectability converges towards the limit. With a telescope of $100 \mathrm{~m}$, we reach $1.5 .10^{-10}$ in one hundred of hours at 0.1 " from the center for both systems.

Another relevant point is that the photon noise depends on the level impressed by the adaptive optics. Indeed, it is included in each direct image before subtraction and, as said in section 3, these images are dominated by the residual of AO.

Thus, beyond static aberrations, the detectability capacity of the ELTs relies on the performance of the AO system. Close to the star, the pyramidal sensor is more performing than the Shack-Hartmann sensor but in the region of interest, this difference becomes low and allow us to gain less than a factor of 2 on the photon noise.

\section{Conclusion}

For this study, we made simple assumptions: the coronagraph is perfect, the pupil is not segmented and has no central obstruction; we chose a quite simple model for the AO system and we took into account static aberrations upstream the coronagraph and downstream to account for the calibration imperfection. In spite of the simplicity, the results are important: to be able to detect an Earth-like planet in the neighborhood (0.1") of a Sun-like star, we have to constrain the static aberrations very strongly, limiting common path aberrations to $5 \mathrm{~nm}$ and differential aberrations to $0.2 \mathrm{~nm}$. Another fundamental limitation comes from the exposure time: the aberrations which limit the detectability in the case of direct imaging, namely residual aberrations after AO, will determine the level of the photon noise. Thus reaching $10^{-10}$ with our hypotheses requires a few hundreds of hours.

The detection of Earth-like planets, even with a telescope of $100 \mathrm{~m}$, is a great challenge and a thorough analysis of the whole system is necessary to determine precisely the capability of detection of such telescopes.

\section{References}

Cavarroc, C., Boccaletti, A., Baudoz, P., Fusco, T. \& Rouan, D. 2005, accepted by A\& $A$

Duparré, A., Ferre-Borrull, J., Gliech, S., Notni, G., Steinert, J., \& Bennett, J. M. 2002, AO 41, 154

Racine, R., Walker, G. A. H., Nadeau, D., Doyon, R., \& Marois, C. 1999, PASP 111, 587

Rigaut, F., Salmon, D., Arsenault, R., Thomas, J., Lai, O., Rouan, D., Véran, J. P., Gigan, P., Crampton, D., Fletcher, J. M., Stilburn, J., Boyer, C., \& Jagourel, P. 1998, PASP 110, 152 